\title{
LES ARBRES DE LA CONNAISSANCE
}

Le précepteur d'Emile, nous répète-t-on, se tromperait deux fois au moins en n'éduquantqu'un seul et solitaire élève, et en ne lui donnant en guise de liberté que la soumission à une nature arrangée. La scène véridique de la pédagogie serait autre un exact apprentissage du réel au sein des collectivités que les maf̂tres d'école organisent. Pourtant celles-ci sont également des paysages. Le présent article évoque trois façons, que trois républiques eurent, de rêver un monde où l'école aurait été possible.

Une lumière qui est celle d'un ciel d'été. Elle montre, aux portes d'une église de village, deux vieux noyers qui penchent leurs feuillages et inclinent leurs troncs. A leurs pieds une source bouillonne, s'élance et aussitôt heurte à des blocs de granit. Inlassablement l'écume lave et blanchit la pierre noire, et dans les rochers fait naître le poli d'un tertre. Un oeil infiniment patient attaché à deviner ce travail un moment s'échapperait. Il fuirait dans la fenêtre formée par le branchage des noyers pour, glissant de colline en colline, regarder au loin bleuir les lacs et ondoyer la plaine. Ignorant ces lointains un désordre d'enfants s'est dans le premier paysage égaillé. A côté des brebis broutant l'herbe du cimetière, de l'hirondelle gobant l'insecte, des passereaux venant émietter le pain qu'on leur lance, des enfants par grappes se sont adossés aux troncs des arbre, juchés sur leurs branches, assis dans le cimetière au pied de la croix ou sur les tertres encore verts des morts du printemps.

Ainsi dispersés ils se tiennent en avant de toute profondeur, fixess sur un même plan par quelque chose au milieu d'eux qui les retient: absolument au centre, mêlée à l'eau de la source, la voix grave du maître d'ecole. Cette représentation d'enfants disposés dans une lumière d'été est représentation d'une école en plein champ"1.

Le poème voudrait, qu'ainsi immobilisés le savoir proche accessible aux sens aussi bien que celui lointain promis à la contemplation, une école puisse dans cette immobilité se tenir. Recueillie et accueillante. Le maître d'ecole

'(Lamartine, Le maître d'école). 
"(...) pense tout haut pour eux; le cercle écoute et (son) coeur dans leurs coeurs se verse goutte à goutte."

Ecoutons. Dans l'eau qui murmure, la voix du maître maintenant parle. Elle dit: Que chaque jour elle déchiffre et épelle, dans la nature, du nom divin quelque lettre nouvelle.

Que dans le grain qui mûrit pour les hommes, et pour les oiseaux aussi, elle fait voir la bonte. Que dans l'ordre naturel elle fait lire la sagesse. Que dans le monde elle fait sentir la grandeur. Peut-être ceux que le poème a rassemblés autour du maître d'école entendent-ils ces choses. Mais en ce jour où le poète élégiaque s'essaie à parler en maître d'ecole, nous entendons aussi, dans ce qu'il dit, le poème qui le dit. Nous entendons le poème séparé de ce qui est dit: le genre didactique. Pendant que le maître d'école pense tout haut au milieu des enfants rassemblés, la forme poétique se contorsionne pour énoncer l'éther, les soleils des autres mondes, les cometes, les orbites planétaires. Insérée au coeur du paysage, mêlee au chant de la source qui polit les plus durs rochers, la voix du maître d'école récite Newton et les Principia mathematica.

Quand le poème qu'une voix récite se fait didactique ${ }^{2}$, le contenu du poème sur lequel la voix se déforme existait déjà. Il existait avant que la voix se soit mise à parler; avant que les enfants se soitent, épars, rassemblés autour d'elle; avant que le paysage où les uns et les autres vont se tenir eât même été formé. Ce contenu, dit Hegel, existait, parfaitement constitué, porté à son état d'achèvement, dans la conscience. Il n'etait aucunement dans la nature qu'on a rassemblée pour l'y faire entendre. Mêlé au murmure de l'eau, il ne suit en fait aucun des trajets de l'eau qui coule et écume entre le bouillonnement natif d'une source et le tertre sur lequel son action s'achève. Il ne ressent aucunement l'appel du spectacle lointain, que la fenêtre de feuillage recèle, des lacs bleuissants et de la plaine ondoyante. Cette vision, ce contact qui dans l'oeil précipite le proche et le lointain, ont été réservés au regard matinal du poète: l'oeil, glissant de colline en colline

"embrasse au matin l'horizon qu'il domine."

${ }^{2} \mathrm{Hegel}$, Esthetique, l'Art symbolique, III. 
Ce moment où la lumière est encore suffisamment tremblante pour qu'effectivement le lac soit vu bleuir, la plaine soit vue ondoyer, où le lac n'a pas encore toute sa couleur, la plaine n'est pas encore entièrement saisie dans sa forme, où le regard encore incertain du poète hesite sur la couleur et la forme dans lesquelles sa vision va fixer les choses, ou son regard entre figures et nuances changeantes se glisse comme vers les choses mêmes; ce moment est rigoureusement antérieur. C'est qu'a l'heure où le poète prend la voix du maître d'ecole, où il fixe l'autre désordre: le désordre d'enfance, dans un paysage sans ombre, les choses ne vacillent plus. Elles ont des contours. Elles ont couleur, forme, grandeur. La lumière d'été fait tomber sur elles une exactitude, qui les fige dans une identité à elles-mêmes que chacun peut fier: "Voyez," dit la voix du maître d'école, "mesurez," "interrogez," "calculez," "comparez."

Pour Hegel, sous la lumière didactique les choses sont aux mains de l'entendement; opérations du regard, de l'outil, de la langue, de la mémoire, du geste, de tout ce que l'on voudra: En leur fond, opérations des mains. Les mains se meuvent et meuvent les choses. Elles travaillent dans le mouvement. Elles décomposent et recomposent de toutes les façons les mouvements auxquels les choses se prêtent sans jamais vaciller. Voir, mesurer, inerroger, calculer, comparer, c'est voir, mesurer, interroger, calculer et comparer l'identité avec elles-memes de choses exactes d'une exactitude antérieure à l'ensemble des opérations, auxquelles la voix du maître d'école invite.

Importe-t-il vraiment que ces enfants ici soient si loin encore, ou trop près déjà, de leurs mains? Que le maître demande des mesures et des calculs à des enfants juchés sur des branches, adossés à des troncs, assis dans la verdure? On sait que des pédagogues feront métier de mettre ces coprs en cohérence avec le travail des mains, ou de rappeler ces mains aux projets des corps. Ici, fixés aus sein d'un paysage premier de la pédagogie, disposés pour l'accueil ou se mouvant dans les choses, corps et mains se separent peut-être moins l'un de l'autre qu'ils émergent encore, ensemble, de l'universelle fluidité. Leur jeunesse ne les a pas beaucoup éloignés de celle du jeune Théêtete, tel qu'en 154c (Platon, Thétete) Socrate, avant de le conduite devant les paysages aporétiques de la science, l'arrachait aux sensations de la fluxtuation de la couleur, de la température, de la grander. C'est à Thétete plein du sentiment de l'instabilite de toutes choses, en effet, que Socrate faisait soudain supposer qu'à portée de sa main il y aurait six osselets alignés, auxquels 
viendraient s'en ajouter quatre puis douze autres; des osselets là, qu'on déplace, qu'on compte, qu'on mesure et qu'on compare à partir des ensembles qu'ils formemt; des choses pour les operations de la main. Mais justement, c'était-ce pas là à nouveau une scène quelque peu étrange? comment expérimenter exactement les mouvements auxquels les osselets se prêtent quand la main qui effectue les mouvements ainsi que les osselets dont elle se saisit sont dans l'entendement? Quand 6, on l'apprendra en $204 c$, se fluidifie en 1 .....6, $2 \times 4,3 \times 2,4+2,3+2$ et peut-être d'autres choses? D'où peut à ces six osselets supposés, à cette main imaginée, venir la stabilité nécessaire à l'exactitude de l'opération? Et si cette exactitude vient d'ailleurs, quel besoin a-t-on ici d'une main, d'osselets? A supposer enfin qu'on confirme ce besoin, l'étrangeté serait-elle vraiment moindre de mettre réllement les osselets dans la main de Théetete?

Plus tard, de fait, les écoliers manipulent les osselets. Ils apprennent les mouvements qui conservent et métamorphosent leurs arrangements, ils écrivent et font jouer les formules qui les représentent. Mais, aux yeux d'un mathématicien qui observe, ils n'en continuent pas moins à faire des choses séparées: à accueillir les contenus de l'entendement et à mouvoir les objets; à s'ouvrir à l'existence logique des nombres et à mettre en mouvement les formules qui les produisent arithmétiquement. L'effort du mathématicien, son effort pédagogique, va consister à conduire ces opérations vers une coincidence: guidée par une méthode, la main de l'écolier est engagée dans une reprise si excate de

\footnotetext{
'tous les motifs et tous les pas qui ont conduit à la recherche et à l'invention des formules, qu'il est impossible de se servir des formules sans que l'esprit repasse sur tous les motifs et sur tous les secrets de leur artifice: alors, l'esprit et la main operent ensemble: tantôt la main precede l'esprit; tantôt l'esprit précede la main: mais jamais ils ne sont éloignés; toujours ils se suivent de près's.
}

Apprendre à compter, apprendre les opérations, ce serait compter et faire des opérations d'une manière telle qu'un observateur ignorerait à chaque instant si l'exactitude est dans la main qui meut les formules ou dans l'esprit qui institue la chose exacte; si le corps et cette main sont en train de manipuler des signes ou de concevoir des idées. La volonté de faire de cette ignorance une méthode est productrice

\footnotetext{
3(Moyens d'apprendre à compter surement et avec facilité, An VII, p. 6).
} 
d'artifices nouveaux, qui ajoutent à la langue la plus anciennement fixée. A la série des nombres que la langue connaît, l'ecole ajoute d'autres combinaisons de signe et de sens susceptibles d'effectuer l'opération numérique. Et elle le fait en particulier là où dans la série des nombres il semble qu'il n'y ait pas cette indécision quant au signe ou au sens, là ou seul le signe accessible à la main semble se présenter. Regardons l'exemple: l'appellation des dizaines. Pour trente, quarante, cinquante, soixante, les mots sont tels que, pour peu qu'on ait fait compter l'ecolier dans un ordre qui lui ait rendu sensible une logique, on ne sait jamais, au momentou il les dit, s'il les répète machinalement ou s'il recompose en esprit la dimension de la dizaine sur les unités. Dans les mots trente, quarante, cinquante, soixante, l'analogie verbale est en effet assez conservee pour qu'on puisse ignorer jusqu'au bout qui, de la mécanique ou de l'esprit, a appelé l'autre sur le chemin qui va de trois à trente, de quatre à quarante, etc.

En revanche,

'dans les mots vingt-quatre, quatre-vingt, quatre-vingt dix le nombre des dizaines dont on parle n'est pas du tout marque par l'analogie des mots: on croirait que ce sont deux langues différentes, que dans l'une on procede par dizaines, trente, quarante, cinquante, soixante, et dans l'autre par vingtaines, ving, quatre-vingts, quatrevingt-dix'".

Sans doute deux langues différentes, voire de multiples langues, laissent-elles autant de chances à l'esprit par rapport au machinal, et d'autres considerations agissent-elles sur le contemporain de la Révolution française pour qu'il propose, avec l'introduction de duante, de septante, d'octante, de nonante, l'unification décimale de la langue française. Mais cela n'empeche peut-être pas l'essentiel ici d'être que l'artifice unificateur en même temps rende indécidable la question du machinal et de l'esprit, dans cette indécidabilité les conjoinge, et nomme cela apprendre.

Peut -être même peut-on rester sensible à l'autre aspect de cette invention pédagogique conservée dans les oeuvres du marquis de Condorcet, et dans le remplacement de vingt par duante ou de soixantedix par septante retrouver le très ancien programme d'exclure de l'univers

\footnotetext{
${ }^{4}$ ibidem, p.8.
} 
nombré la démesure. L'exactitude de duante, exacte expression de 20 dans un système décimal, s'oppose à l'excroissance de vingt sur la série décuple. Identifiant dans le rejeton de vinginti une figure du surplomb, dans sa face effacée par delà l'usure des siècles discernant encore ses prestiges passés, éprouvant les liens mythiques qui auraient uni sa saillie à des despotismes antiques ${ }^{5}$, duante rêverait le déploiement d'une culture qui s'entiendrait au contraire aux rapports exacts entre les choses.

\begin{abstract}
'Ces attentions, ecrit l'inventeur de duante, pourront paraitre minutieuses; mais ce sera à ceux qui ignorent que l'analogie des mots et des idées est le fil tantot visible, tantot invisible, qui a guidé les hommes de genie et les peuples dans la creation et dans le progrès de tous les arts, de toutes les sciences.".
\end{abstract}

Créer duante à la place de vingt ou dullion à la place de milliard, conduire à un système entier d'art et de science la volonté de ne rien devoir à l'exception, de laisser le moins possible à l'incommensurable, ce que sur le chemin des langues mortes apprises dans les colleges ces gestes pour leur compte retrouveraient en guise d'échose au logos grec ou à la mousiké pythagoricienne', que d'autres gestes recueilleraient éventuellement autrement, serait le projet d'une culture d'hommes libres. Lire, écrire, compter, en partie reçus du pythagorisme et parfois vibrant encore de son exacte tension seraient de cela la formule. Peut-être. Encore que les uns pourraient entendre, dans l'écho qu'octante (contre huitante) fait à octidi, octogenaire et octave, trop de mélodie dans une matière où seule l'exactitude devrait compter. A nouveau, importe-t-il vraiment? Dans le labyrinthe du lire-écrire-compter on peut peut-être se laisser guider par le fil du chant oublié aussi bien que par celui de la ratio promise. Tous deux présentent une alternance de visibilité et d'invisibilité et c'est elle qui est d'abord releveee. Peut-être parce qu'une culture qui propose autre chose que de reproduire la démesure se gagnerait dans ce clignotement.

Néanmoins, regardons encore le paysage scolaire. Au moment où le territoire français se quadrille d'écoles et de maître, les chênes

\footnotetext{
${ }^{5} \mathrm{~A}$ E Kane, Systèmes de numération dans quelques pays d'Afrique de l'Ouest, (thèse de l'Université de Lille).

'Ibidem.

'J. Lohmann, Mousike et logos, Ed. fr. TER, 1989.
} 
deviennent des arbres qui meurent. Leurs figures imposantes, que le souvenir aussitôt s'empresse d'imager dans des forêts ancestrales et des bois sacrés bravant les siècles, défiant les cieux, dominant la terre, tombent sous les coups de l'age, de la foudre et des hommes; et dans leur chute rendent visible la chute d'anciennes formes de vie. S'imposent des essences moins altières, plus jeunes, plus régionales: pins et arbousiers dans les Hautes Pyrénées, saules dans le Cher, cyprès et vergers dans le Doubs, pommiers en Charente, peupliers dans l'Aude, noisetiers dans les Alpes, chataigniers dans l'Ariege, sapins dans le Jura ${ }^{8}$

Paysages taillés sur mesure pour évoquer les nouvelles images de la vie, écosystemes de petits mammiferes, oiseaux ou insectes, cris et jeux d'enfants, sorties familiales, solitudes d'aquarellistes. Or dans ces taillis, dont justement la promesse d'enchantements pour chacun une dernière fois salue les enchantements congédiés qui composaient le merveilleux de la "haute forêt" par la geste d'Artus, l'enseignant se laisse mal representer.

'Plus complete qu'aucune autre des beautés de la nature la forêt, explique-t-on en effet aux écoliers, offre à chacun ce qu'il desire: au reveur un aliment pour ses songes, a l'observateur mille sujets d'étude, au peintre toutes les harmonies de la lumière et de la couleur, a ceux qui cherchent le mouvement et le déploiement de la force physique elle donne le plaisir de la chasse et des longues courses. Les enfants y sont amenes à la fois par l'attrait des libres promenades et par les manifestations si intense et si diverses de la vie?."

L'instructif récit qui suit cette annonce dès lors chacun trouvant à s'y enchanter, sauf le maître d'ecole, en l'occurrence une institutrice que des stances à la forêt déclamées par les taillis et les chemins aux heures tristes du crepuscule, et des leçons versifiees faites aux enfants du village, conduisent séance tenante à l'asile d'aliénés du département. Bien sûr pour cette institutrice aussi un conte pourra continuer à s'accomplir sous les arbres, la bonté éclairee d'une famille moderne habitante de la forêt aux vacances d'été la délivrer de son sort et lui faire épouser le fils héritier du château. Mais ce mariage, réunissant les promis de tout au bout d'un chemin entièrement fraye à travers des lettres, des journaux,

\footnotetext{
${ }^{8}$ M. Besson et M. Abadie, Anthologie des instituteurs-poètes, (Paris: Bibliothèque de l'association, s.d.).

${ }^{9}$ E. D. Labesse et H. Pierret; Promenades en forêt, p. 4.
} 
des actes médicaux, des enquêtes administratives, trop tard accompli, célébré post mortem, porte le deuil précisément des mariages auxquels un vent léger accorde le don de se dire dans la langue édémique des arbres qui s'inclinent et des feuillages qui attirent.

"Le très ancien rite des noces du langage avec l'arbre (...) dispensant aussitot de par le monde entier les enfants"

s'accomplit lui sous des arbres séparés de la connaissance. Et peut-être à certaines heures un marcheur, s'arrêtant sous un arbre sans s'arrêter à savoir

"si c'est un auine ou un peuplier"11.

peut-il se laisser frapper tout à coup par ce langage. Enseigner la pédagogie à l'école normale de Rodez plutôt que marcher, cela consiste au contraire à ne cesser de savoir que l'arbre est un peuplier et qu'il est de la nature des peupliers de dessiner, presque désespérément, un la nuit sombre.

"chemin (qui) s'êtend toujours blanc, dès l'éveil du matin radieux jusqua

Une lumière crue, aveuglante, toujours

De l'aube jusqu'au soir les étreint, les inonde;

Ils ont, quand vient midi, de longs silences lourds ${ }^{12}$."

Au pied de cette immobilité, la capacité des pédagogues à faire vibrer l'exactitude elle-même se révèle précieuse.

STEPHANE DOUAILLER

L'exposé ci-dessus a été présenté au Colloque de La Rochelle, "Le métier d'instruire," organisé sous la direction de Laurence Cornu avec le concours du Collège international de philosophie, du 15 au 16 mai 1990.

\footnotetext{
${ }^{10} \mathrm{~W}$. Benjamin, Brèves ombres, l'arbre et le langage.

${ }^{11}$ Ibidem.

${ }^{12}$ A. Lavergne, Parole d'amour, in M. Besson et M. Abadie, op. cit., p. 164.
} 